\title{
DRC Is Not Ready for Transition from Anonymous Unlinked Sero-surveillance to Using PMTCT Program Data for HIV Surveillance, According to the WHO Criteria Assessment Results
}

\author{
Jack Hyyombo Tambwe Kokolomami ${ }^{1,2,}$, , John Shungu Okende ${ }^{2}$, Placide Okitayemba Welo ${ }^{3}$, \\ Felly Isumeliko Ekofo $^{3}$, Etienne Mutombo Mpoyi ${ }^{4}$, Patrick Kalambayi Kayembe ${ }^{1}$ \\ ${ }^{1}$ Department of Epidemiology and Biostatistics, Kinshasa School of Public Health, University of Kinshasa, Kinshasa, DRC \\ ${ }^{2}$ Department of Research, Monitoring and Evaluation, Health Services Development Fund, Kinshasa, DRC \\ ${ }^{3}$ National Program Against HIV/AIDS, Ministry of Public Health, Kinshasa, DRC \\ ${ }^{4}$ World Health Organization Bureau for DRC, Kinshasa, DRC
}

Email address:

pajack70@yahoo.fr (J. H. T. Kokolomami)

${ }^{*}$ Corresponding author

\section{To cite this article:}

Jack Hyyombo Tambwe Kokolomami, John Shungu Okende, Placide Okitayemba Welo, Felly Isumeliko Ekofo, Etienne Mutombo Mpoyi, Patrick Kalambayi Kayembe. DRC Is Not Ready for Transition from Anonymous Unlinked Sero-surveillance to Using PMTCT Program Data for HIV Surveillance, According to the WHO Criteria Assessment Results. Central African Journal of Public Health. Vol. 4, No. 5, 2018, pp. 143-148. doi: 10.11648/j.cajph.20180405.13

Received: August 28, 2018; Accepted: September 6, 2018; Published: October 10, 2018

\begin{abstract}
Since 2003, the Democratic Republic of Congo (DRC) has carried on HIV surveillance through Anonymous Unlinked Testing in Sentinel Surveillance sites (AUT-SS) amongst pregnant women attending Antenatal Clinics (ANC). However, this expensive process raises ethical limitations. In 2013, the World Health Organization (WHO) recommended countries to evaluate the possibility of transitioning from the AUT-SS to surveillance based on data from the routine diagnosis of HIV in Prevention of Mother-To-Child Transmission (PMTCT) programs. The authors carried out this evaluation for DRC. An evaluation of four of the five criteria proposed by the WHO was conducted by following the defined procedure, and comparing with the determined benchmarks, to assess whether DRC can undergo the transition. Data for this assessment were collected in 2013 and 2015 during sentinel surveillance activities. AUT- SS data of 26,301 pregnant women were collected from 60 sentinel sites, while data from PMTCT routine testing were available for 12,291 women. The overall rate of agreement between routine PMTCT and AUT-SS test results (Cohen's Kappa coefficient of agreement) was $0.7 \%$ (95\% CI: $0.65-0.76 \%$ ), the proportion of positive agreement (PPA) was $65.7 \%$ (95\% CI: 59.8\%-71.1\%), and the proportion of negative agreement (PNA) was 99.5\% (95\% CI: 99.4\%-99.7\%). The level of non-consent bias in the routine screening test was 4.8\%, with a ratio of difference in prevalence of 1 to 10. Eighty-three per cent of sentinel surveillance sites also offered PMTCT service, which was discontinued for $34 \%$ of them. DRC must still strengthen the robustness of routine HIV testing, the overall rate of access to this test, its permanence/regularity, and the quality of the data collected in routine, before claiming to use the data from the PMTCT program for purposes of HIV surveillance, in lieu of the ANC AUT- SS.
\end{abstract}

Keywords: HIV Surveillance, Anonymous Unlinked Test, Prevention of Mother-To-Child Transmission, World Health Organization Criteria Assessment, DRC

\section{Introduction}

In 2013, the HIV prevalence in the general population of the Democratic Republic of Congo (DRC) was 1.2\% [1]. In accordance with the World Health Organization guidelines formalized in 2003, sentinel surveillance (SS) of HIV infection among pregnant women attending antenatal clinics 
(ANC), through an anonymous and unlinked testing (AUT), can be used to estimate the magnitude of such an epidemic, and the tendency of its key indicators [2,3]. However, the use of the ANC and AUT-SS data has some limitations. One of them is ethical: tested women neither give their consent, nor have access to the result of the test, which, if infection is revealed, should lead to their discharge under antiretroviral therapy (ART) for the benefit of the mother and the child. The other limitation is the mobilization of huge human, material, and financial resources needed for the implementation of such activities [4-6]. Since 2003, the HIV epidemic sentinel surveillance field activities are organized every two years, although the protocol calls for a yearly collection of data. For a country as large as the DRC, the needful funding for annual SS passages may not be covered by domestic funds, so that the years where external financing failed, the SS had not been conducted. The DRC could therefore only be interested in the current WHO recommendations to use as far as possible routine HIV screening data, including and especially those from the prevention of mother-to-child transmission of HIV programs (PMTCT), for purposes of HIV surveillance. Before transitioning from ANC AUT-SS data based surveillance to the one using routine testing on PMTCT program data, it should be assessed whether the country is ready to do this. The WHO recommends to evaluate five criteria of the database and the quality of the PMTCT program data for this assessment [7]. This work evaluated four of these five criteria.

\section{Methods}

Data from 2013 and 2015 ANC AUT-SS HIV surveillance in DRC were used to extract four of the five WHO criteria.

In 2013, the ANC AUT-SS was conducted in 50 sentinel sites, of which 14 were already offering routine HIV testing services for at least two years. In each of these 14 sites, among the women eligible for the ANC and AUT-SS, and who had also consented and received HIV tests in PMTCT services, three records (four for Kinshasa sites), have been randomly selected to collect the information needed in the evaluation of the PMTCT data quality. This collection was made from a questionnaire developed in accordance with the one provided by the WHO and United Nations program on HIV/AIDS (UNAIDS).

In 2015, the country extended ANC AUT-SS activities to 60 sentinel sites, of which 23 were rural. Of these 60 sentinel sites, 50 also offered routine HIV tests in PMTCT services. In accordance with the current protocol, the ANC AUT-SS tests were anonymous and unlinked. Pregnant women aged 15 to 49 years seeking antenatal care in one of the 60 clinics selected as sentinel sites, and who were at their first visit for current pregnancy, were sampled in a consecutive way from September $9^{\text {th }}$ to $30^{\text {th }}$, or until the minimum number of 400 women per site was achieved. A short questionnaire was used to collect demographic and behavioral data, as well as the medical history of each participant. Then, for syphilis screening, blood samples were collected $(5$ to $10 \mathrm{ml})$ in evacuated tubes [Vacutainer, Becton, Dickinson \& Company, Rutherford, NJ 07070], which were left on the bench until coagulation or centrifuged to obtain serum. Each participant was informed of the syphilis screening result and appropriate free treatment was provided when needed. Subsequently, any identity connection between the participant and her blood sample was removed, making the data in the questionnaire and the blood sample anonymous and unlinked. Aliquots of the sample were then extracted for HIV testing. The HIV test was performed using the algorithm of SS which uses up to three rapid tests of third generation: (1) HIV-1/-2 (Alere Medical Co., Ltd., Japan, 100 tests kit, REF. 7D2343) as a screening tests. Non-reactive samples were considered as HIV negative. Reactive samples were submitted at the same time to (2) Unigold HIV-1/-2 (Trinity Biotech plc, Bray, Ireland, 20 tests kit, Cat. No. 1206502), and (3) Double Check Gold HIV-1/-2 (Invesness Medical Innovations, Orgenics Ltd., REF 70635100). If Unigold HIV-1/-2 and Double Check Gold HIV-1/-2 tests were both reactive, the sample was classified as HIV positive. On the other hand, if both were non-reactive, the sample was recorded as HIV negative. In case Unigold $\mathrm{HIV}-1 /-2$ had been reactive and Double Check Gold HIV-1/-2 non-reactive, then the result was recorded as indeterminate. If the Unigold HIV-1/-2 test was non-reactive and Double Check Gold HIV-1/-2 was reactive, the result was recorded as presumptuously positive. Samples with indeterminate and presumptuously positive results were tested using ELISA algorithm at the National Reference HIV laboratory (LNRS), and the result of this last test was retained. A quality assurance and quality control system was established to validate the field (site) test results: all HIV positive samples and $10 \%$ of negative samples have been tested at LNRS according to the ELISA HIV algorithm (Vironostika HIV Ag/Ab, Microelisa system, Bio Merieux, REF 259852 and Enzygnost Anti-HIV Plus, 192 tests kit, Siemens Health Care, REF OQFK 13 and INNOLIA HIV $1 / 2$ Score, INNOGENETICS, 20 tests kit, REF 80540). According to the protocol, for the sites for which the discordance in the results between the field and the LNRS would reach 10 per cent, all samples should be tested again at the LNRS (this case has not been observed in 2015).

The WHO criteria for the use of the data from the PMTCT for surveillance purposes were assessed as follows:

(a) The evaluation of agreement at the individual level between the ANC AUT - SS HIV test and the PMTCT routine test results was made by calculating the Cohen's Kappa coefficient of concordance, the proportion of positive agreement (PPA) and the proportion of negative agreement (PNA). These calculations have been made in accordance with the WHO guidelines, considering the result of the AUT test as reference, and the results were compared to the WHO benchmarks.

(b) The measurement of selection bias in the practice of routine HIV test in PMTCT was made by calculating the magnitude of the bias, and also its direction. The direction of bias has been evaluated by the combined 
calculation of the ratio of difference in HIV prevalence among pregnant women received in ANC-SS who underwent routine PMTCT HIV testing, and those who have not received it; and the overall rate of access to the routine PMTCT service of all women received in ANC-SS. The results of calculations have been compared to standards and interpreted in accordance with the WHO recommendations.

(c) The completeness in the routine HIV testing in PMTCT of the ANC-SS sites has been evaluated at two levels: at the site level (proportion of ANC- SS sites that also offer the routine HIV test as part of the PMTCT service) and at the individual level (proportion of women who had access to the PMTCT-based HIV testing service among all women tested in ANC- SS sites).

(d) The quality of data collected on routine basis in PMTCT was assessed by considering as a whole the 49 records collected in the only 14 sites that met the criterion of offering the routine HIV test for at least 2 years during the ANC-SS activities in 2013. From the WHO model questionnaire used to collect the data, 7 key elements were extracted (the age of the woman, the offer of HIV testing in routine, the acceptance or not of this test, the test date, the result of this HIV test, the offer of syphilis test, and the result of the syphilis test), and used a twice $90 \%$ rule to judge : an element was considered to be well recorded if it was not missing in at least $90 \%$ of records, that is 44 of the 49 records in all of the routine ANC registers, and the quality of data was considered good if at least $90 \%$ of these key elements (at least 6 items on 7) have been well recorded.

(e) In 2015, data on the quality of routine PMTCT HIV tests were collected in 9 of the 50 sites, all chosen for convenience. It was considered that data did not adequately represent the 50 sites, as the evaluation process had not been standardized in all 9 sites as well. Therefore, with these data, it was not possible to assess the quality of routine HIV testing in PMTCT, neither at the site level, nor at the collective level. It is the only one of the five criteria of the WHO assessment recommendations which could not been assessed.

\section{Results}

The ANC and AUT-SS data were available for 26,301 pregnant women, collected at 60 sentinel sites, while data from PMTCT routine testing were available for 12,291women.

Table 1. Positivity proportion of HIV test among pregnant women attending ANC, from AUT at sentinel surveillance sites and from ANC routine registers records in DRC, 2015.

\begin{tabular}{|c|c|c|c|c|c|c|c|}
\hline \multirow{3}{*}{ Variable } & \multirow{3}{*}{ Modalities } & \multirow{2}{*}{\multicolumn{2}{|c|}{$\begin{array}{l}\text { ANC AUT-SS } \\
\text { Number }\end{array}$}} & \multicolumn{4}{|c|}{ ANC routine registers records } \\
\hline & & & & \multirow{2}{*}{$\begin{array}{l}\text { Positive results } \\
\text { proportion }\end{array}$} & \multicolumn{2}{|c|}{ Number } & \multirow{2}{*}{$\begin{array}{l}\text { Positive results } \\
\text { proportion }\end{array}$} \\
\hline & & Positive & Total tested & & Positive & Total tested & \\
\hline \multirow{8}{*}{$\begin{array}{l}\text { Woman's Age } \\
\text { (years) }\end{array}$} & $15-19$ & 76 & 6040 & $1.3 \%$ & 42 & 2633 & $1.6 \%$ \\
\hline & $20-24$ & 136 & 7138 & $1.9 \%$ & 56 & 3357 & $1.7 \%$ \\
\hline & $15-24$ & 212 & 13178 & $1.6 \%$ & 98 & 5990 & $1.6 \%$ \\
\hline & $25-29$ & 149 & 5915 & $2.5 \%$ & 52 & 2914 & $1.8 \%$ \\
\hline & $30-34$ & 110 & 4089 & $2.7 \%$ & 46 & 1910 & $2.4 \%$ \\
\hline & $35-39$ & 51 & 2249 & $2.3 \%$ & 26 & 1086 & $2.4 \%$ \\
\hline & $40-44$ & 20 & 783 & $2.6 \%$ & 7 & 364 & $1.9 \%$ \\
\hline & $45-49$ & 4 & 87 & $4.6 \%$ & 2 & 27 & $7.4 \%$ \\
\hline \multirow{4}{*}{ Marital status } & Single & 47 & 1843 & $2.6 \%$ & 27 & 893 & $3.0 \%$ \\
\hline & Married & 472 & 24036 & $2.0 \%$ & 194 & 11235 & $1.7 \%$ \\
\hline & Separated/Divorced & 16 & 348 & $4.6 \%$ & 6 & 133 & $4.5 \%$ \\
\hline & Widowed & 11 & 74 & $14.9 \%$ & 4 & 30 & $13.3 \%$ \\
\hline \multirow{4}{*}{ Instruction } & $\begin{array}{l}\text { No formal } \\
\text { instruction }\end{array}$ & 33 & 1492 & $2.2 \%$ & 15 & 490 & $3.1 \%$ \\
\hline & Primary school & 172 & 7467 & $2.3 \%$ & 69 & 2824 & $2.4 \%$ \\
\hline & High school & 328 & 16347 & $2.0 \%$ & 142 & 8342 & $1.7 \%$ \\
\hline & $\begin{array}{l}\text { Higher/Superior/ } \\
\text { University education }\end{array}$ & 13 & 995 & $1.3 \%$ & 5 & 635 & $.8 \%$ \\
\hline \multirow{10}{*}{$\begin{array}{l}\text { Number of } \\
\text { pregnancies } \\
\text { carried } \\
\text { including } \\
\text { current }\end{array}$} & One & 98 & 6277 & $1.6 \%$ & 49 & 3105 & $1.6 \%$ \\
\hline & Two & 66 & 4401 & $1.5 \%$ & 30 & 2171 & $1.4 \%$ \\
\hline & Three & 100 & 4181 & $2.4 \%$ & 46 & 1991 & $2.3 \%$ \\
\hline & Four & 109 & 3354 & $3.2 \%$ & 37 & 1548 & $2.4 \%$ \\
\hline & Five & 61 & 2650 & $2.3 \%$ & 23 & 1161 & $2.0 \%$ \\
\hline & Six & 40 & 1941 & $2.1 \%$ & 14 & 858 & $1.6 \%$ \\
\hline & Seven & 35 & 1437 & $2.4 \%$ & 19 & 591 & $3.2 \%$ \\
\hline & Eight & 15 & 871 & $1.7 \%$ & 6 & 374 & $1.6 \%$ \\
\hline & Nine & 10 & 539 & $1.9 \%$ & 4 & 225 & $1.8 \%$ \\
\hline & Ten and more & 12 & 650 & $1.8 \%$ & 3 & 267 & $1.1 \%$ \\
\hline
\end{tabular}




\begin{tabular}{|c|c|c|c|c|c|c|c|}
\hline \multirow{3}{*}{ Variable } & \multirow{3}{*}{ Modalities } & \multirow{2}{*}{\multicolumn{2}{|c|}{$\begin{array}{l}\text { ANC AUT-SS } \\
\text { Number }\end{array}$}} & \multicolumn{4}{|c|}{ ANC routine registers records } \\
\hline & & & & \multirow{2}{*}{$\begin{array}{l}\text { Positive results } \\
\text { proportion }\end{array}$} & \multicolumn{2}{|c|}{ Number } & \multirow{2}{*}{$\begin{array}{l}\text { Positive results } \\
\text { proportion }\end{array}$} \\
\hline & & Positive & Total tested & & Positive & Total tested & \\
\hline \multirow{2}{*}{$\begin{array}{l}\text { Living } \\
\text { environment }\end{array}$} & Rural & 342 & 16231 & $2.1 \%$ & 122 & 5111 & $2.4 \%$ \\
\hline & Urban & 204 & 10070 & $2.0 \%$ & 109 & 7180 & $1.5 \%$ \\
\hline \multirow{2}{*}{$\begin{array}{l}\text { Syphilis test } \\
\text { result }\end{array}$} & Positive & 16 & 325 & $4.9 \%$ & 8 & 104 & $7.7 \%$ \\
\hline & Negative & 530 & 25976 & $2.0 \%$ & 223 & 12187 & $1.8 \%$ \\
\hline
\end{tabular}

In ANC-SS as in routine PMTCT, the HIV test positivity proportion for women aged 15 to 24 years is significantly less than that of all other age groups (multiple comparisons results not shown). In ANC-SS data, the HIV test positivity proportion is not significantly different between married women and single ones, while for routine data the first is significantly less than the second, as it is in comparison with each of all the other categories of marital status. On the other hand, the level of education of women does not appear to be associated with the HIV test results, either in ANC-SS or in routine PMTCT.

WHO criteria assessment:

\subsection{Assessment of the First Criterion: Agreement Between ANC Sentinel Surveillance and Routine PMTCT HIV Test Results}

A total of 12,291 pregnant women were tested on a routine basis in PMTCT (consent obtained and test materials available) among the 26,301 who were received in ANC-SS. From them, among the 268 ANC AUT-SS test positive pregnant women, 92 tested negative by the routine test in PMTCT. Among the 12,023 women registered as negative to the test at ANC AUT-SS, 55 were tested positive to the routine test at PMTCT. Based on these data, the overall agreement rate (Cohen's Kappa coefficient) was $0.7 \%(95 \%$ CI: $0.65-0.76 \%$ ). The proportion of positive agreement (PPA) was $65.7 \%$ (95\% CI: $59.8-71.1 \%$ ), and the proportion of negative agreement (PNA) was 99.5\% (95\% CI: 99.4 $99.7 \%)$.

\subsection{Assessment of the Second Criterion: Magnitude of Non-consent Bias in Routine Antenatal Testing}

Among the 12,291 pregnant women who received PMTCT HIV routine testing, $2.18 \%$

(95\% CI: $1.93-2.45 \%$ ) were positive with the HIV ANC-SS test, as were $1.98 \%$ (95\% CI: $1.76-2.23 \%)$ of the 14,010 women enrolled in ANC - SS who did not get routine test. In comparison, $546(2.08 \%)$ of the total of 26,301 pregnant women tested in ANC AUT-SS had a positive HIV test result. Those figures correspond to a bias rate (magnitude) of $+4.81 \%$ and a ratio of difference in prevalence of 1.1. The overall rate of access to the routine PMTCT services among women tested in ANC-SS was $46.73 \%$.

\subsection{Assessment of the Third Criterion: Offer of Routine PMTCT Services in the ANC-SS Sites}

Of the 60 ANC-SS sites visited in 2015, 50 sites (or $83.33 \%$ ) also offered routine PMTCT service. However, the service was not available permanently in the majority of cases. One third of these 50 sites (34\%) could offer the HIV test to less than half of women attending the ANC-SS. When the HIV test was proposed, the proportion of acceptance by pregnant women was $95.9 \%$.

\subsection{Assessment of the Fourth Criterion: PMTCT Data Quality}

This criterion could not be evaluated using data collected in 2015, as no site assessment had been made that year. From data collected in 2013 in the only 14 ANC sites that met the criterion of having integrated PMTCT for at least 2 years, the table 2 below has been constructed.

Table 2. Assessment of some criteria on the suitability of routine ANC records in DRC, 2013.

\begin{tabular}{lll}
\hline Criterion & $\begin{array}{l}\text { Number of records } \\
\text { assessed }\end{array}$ & $\begin{array}{l}\text { Number of records in which information could be } \\
\text { found in at least one routine register }\end{array}$ \\
\hline 1 Record of the woman's age & 49 & 49 \\
2 Record of whether HIV testing has been offered & 49 & 48 \\
3 Record of whether the HIV test has been accepted & 49 & 48 \\
4 Record of the date of the HIV test & 49 & 48 \\
5 Record of the HIV test result & 49 & 49 \\
6 Record of the date of the syphilis test & 49 & 43 \\
7 Record of the syphilis test result & 49 & 44.0 \\
\hline
\end{tabular}


For two of the seven key elements, namely the mention of the date of screening for syphilis in routine and the mention of the result of this test, the information appeared in at least one of the four routines registers consulted only in $87.8 \%$ and $89.8 \%$ of records respectively. Routine records then reported appropriately the information (mentioned in more than $90 \%$ of cases) of 5 of the 7 evaluated elements (71.4\%).

\subsection{Quality of the Laboratory Test for HIV Screening}

This fifth WHO criterion could not be evaluated due to lack of reliable data.

\section{Discussion}

Subsequent to assessing the five criteria proposed by the WHO, some researchers have shown that some countries were ready to transit from ANC-SS data to the routine PMTCT data, for HIV surveillance purpose [8].

As regards the $\mathrm{DRC}$, on the four evaluated criteria, it was found that:

(1) The agreement between the results of routine HIV testing and the ANC AUT-SS test is low: the overall agreement rate was $70 \%$ (95\% CI: $65 \%-75 \%$ ), the proportion of positive agreement (PPA) and the proportion of negative agreement (PNA) were respectively of $65.7 \%$ (95\% CI: 59.8-71.1\%) and 99.5\% (95\% CI: 99.4 - 99.6\%). These agreement levels are below the WHO benchmarks which are $97 \%$ and $99.9 \%$ respectively for the PPA and PNA, given the level of the ANC prevalence in the country [7]. Therefore, this WHO criterion is not yet fulfilled. The low level of the PPA is particularly worrying. Indeed, taking the result of the ANC AUT-SS test as reference, 34.4 per cent of HIV positive pregnant women would be misclassified (tested negative) in the routine testing. This has the double disadvantage of underestimating the prevalence of HIV among pregnant women when measured by routine PMTCT testing, and reducing the effectiveness of the PMTCT program in the vision of eliminating the mother-to-child HIV transmission. Compliance with standard procedures in executing HIV screening tests, crucial to ensure quality, is often partial in developing countries, leading to a low agreement rate. In Mozambique, a similar study found the proportion of overall positive agreement between the results of routine HIV testing and the results of AUT to be $88.5 \%$ (95\% IC: $85,7 \%-91,3 \%)$ and the proportion of negative agreement to be $98,9 \%$ (95\% IC: $98,5 \%$ $99,1 \%$ ). This low agreement level, in comparison to the WHO benchmarks, has led the country to delay the transition till the standard of PMTCT data has improved before moving to the HIV surveillance based on PMTCT routine data [9]. Kenya, Zimbabwe, and Brazil, facing the same reality, took similar decisions [10-12].

(2) The level of non-consent bias in the routine screening test is $+4.8 \%$. This figure is in accordance with the WHO benchmarks $(-10 \%$ to $+10 \%)$. However, even with a ratio of difference in prevalence of 1.1 , the overall rate of access to the routine PMTCT services among women tested in ANC AUT-SS is $46.73 \%$ (far from the WHO benchmark that is at least $90 \%$ ). The effect of reducing selection bias by improving access to routine services testing was missing, which led to the fact that this criterion was not completely filled. A low level of bias combined with a high rate of use of PMTCT services, when other criteria are met, would put the country in a comfortable position to transit to the HIV surveillance using programmatic data from routine PMTCT services. Zimbabwe, for example, combines a level of non-consent bias of $-2.6 \%$ with a rate of use of PMTCT services of $95.2 \%$ [11].

(3) Only 50 ANC-SS sites on the $60(83.3 \%)$ offered the routine PMTCT services. Although this figure is similar to that reported in some bordering countries such as Cameroon in 2017 [13] and other countries of the sub-Saharan region [14], it is still below the $100 \%$ which is the desired standard. In addition, 34\% of ANC-SS sites delivering PMTCT services were only able to provide intermittent HIV testing (data not shown). Frequent unavailable biological tests and other essential materials could explain the intermittent offer of HIV screening as it has been described elsewhere [15]. Moreover, over half of the sites offering HIV routine testing started this service less than two years ago.

(4) The quality of routine data in ANC-SS registers still needs to be improved. Indeed, on the basis of the criterion of judgment of twice $90 \%$ that have been developed and explained above, and due to the fact that for two of the seven assessed criteria, the information was mentioned in the routine ANC-SS registers in less than $90 \%$ of cases, the quality of routine ANC registers data (including PMTCT registers data) cannot be considered as satisfactory. It is worrying to notice that the clinical registers show a paucity of recorded information linked to the routine screening test for syphilis. This could be an indication that the clinical providers report more frequently and correctly information related to a new activity with dedicated supervision and monitoring, and neglect related information already entered into routine activities. A similar situation might exist in the release of information about the routine HIV testing and results.

\section{Conclusion}

The DRC experiences a low level of non-consent bias in HIV routine testing. However, the country must reinforce the overall access rate to that HIV routine test, its permanence (at least its regularity), the respect of clinical and laboratory 
standards and procedures of testing, and the quality of the collected data, before deciding to use the routine ANC data, and more specifically those of the PMTCT program, for HIV epidemic surveillance as an alternative to the ANC AUT-SS.

\section{References}

[1] Jack Hyyombo Tambwe Kokolomami, Patrick Kalambayi Kayembe. HIV / AIDS Epidemic in the Democratic Republic of the Congo: Current Level of Key Indicators and Projection by 2030. Central African Journal of Public Health. Vol. 4, No. 3, 2018, pp. 86-94. doi: 10.11648/j.cajph.20180403.16.

[2] Joint United Nations Programme on HIV/AIDS \& World Health Organization. Guidelines for conducting HIV sentinel serosurveys among pregnant women and other groups. HIV/AIDS JUNP on, Organization WH, editors. Geneva: UNAIDS; 2003. 66 pp.

[3] World Health Organization. Guidelines for second generation HIV surveillance: the next decade. UNAIDS/WHO Working group on global HIV/AIDS and STI surveillance. Geneva, Switzerland; 2000.

[4] World Health Organization. Conducting HIV Surveillance Based on Routine Programme Data Among Pregnant Women Attending Antenatal Clinics. 2015;(August):88.

[5] Mirkuzie AH, Sisay MM, Hinderaker SG, Moland KM, Mørkve O. Comparing HIV prevalence estimates from prevention of mother-to-child HIV transmission programme and the antenatal HIV surveillance in Addis Ababa. 2012.

[6] Bolu O, Anand A, Swartzendruber A, Hladik W, Marum LH, Sheikh AA, et al. Utility of antenatal HIV surveillance data to evaluate prevention of mother-to-child HIV transmission programs in resource-limited settings. Am J Obstet Gynecol. 2007;197(3 SUPPL.).

[7] UNAIDS/WHO Working Group on Global HIV/AIDS and STI Surveillance. Guidelines for assessing the utility of data from prevention of mother-to-child transmission (PMTCT) programmes for HIV sentinel surveillance among pregnant women. 2013;52. Available from: http://apps.who.int/iris/bitstream/10665/85512/1/9789241505 611_eng.pdf.

[8] Balisanga H, Mutagoma M, Remera E, Kayitesi C, Kayirangwa E, Dee J, et al. HIV surveillance in Rwanda: readiness assessment to transition from antenatal care-based to prevention of mother-to-child transmission program-based HIV surveillance. Int J Infect Dis [Internet]. 2016;52:62-7. Available from: http://dx.doi.org/10.1016/j.ijid.2016.08.029.
[9] Young PW, Mahomed M, Horth RZ, Shiraishi RW, Jani I V. Routine data from prevention of mother-to-child transmission (PMTCT) HIV testing not yet ready for HIV surveillance in Mozambique: a retrospective analysis of matched test results. BMC Infect Dis [Internet]. 2013;13(1):96. Available from: http://bmcinfectdis.biomedcentral.com/articles/10.1186/14712334-13-96.

[10] Sirengo M, Rutherford GW, Otieno-Nyunya B, Kellogg TA, Kimanga D, Muraguri N, et al. Evaluation of Kenya's readiness to transition from sentinel surveillance to routine HIV testing for antenatal clinic-based HIV surveillance. BMC Infect Dis [Internet]. 2016;16(1):1-6. Available from: http://dx.doi.org/10.1186/s12879-016-1434-1.

[11] Gonese E, Mushavi A, Mungati M, Mhangara M, Dzangare J, Mugurungi $\mathrm{O}$, et al. Is Zimbabwe ready to transition from anonymous unlinked sero-surveillance to using prevention of mother to child transmission of HIV (PMTCT) program data for HIV surveillance?: Results of PMTCT utility study, 2012. BMC Infect Dis [Internet]. 2016;16(1):1-13. Available from: http://dx.doi.org/10.1186/s12879-016-1425-2.

[12] Pereira GFM, Sabidó M, Caruso A, Benzaken AS. Transitioning from antenatal surveillance surveys to routine HIV testing: a turning point in the mother-to-child transmission prevention programme for HIV surveillance in Brazil. BMC Infect Dis [Internet]. 2017;17(1):469. Available from: http://bmcinfectdis.biomedcentral.com/articles/10.1186/s1287 9-017-2540-4.

[13] Billong SC, Dee J, Fokam J, Nguefack-Tsague G, Ekali GL, Fodjo R, et al. Feasibility Study of HIV Sentinel Surveillance using PMTCT data in Cameroon: From Scientific Success to Programmatic Failure. BMC Infect Dis [Internet]. 2017;17(1):1-11. Available from: http://dx.doi.org/10.1186/s12879-016-2119-5.

[14] Tudor Car L, Brusamento S, Elmoniry H, van Velthoven MHMMT, Pape UJ, Welch V, et al. The uptake of integrated perinatal prevention of mother-to-child HIV transmission programs in low- and middle-income countries: a systematic review. PLoS One [Internet]. 2013;8(3):e56550. Available from:

http://www.pubmedcentral.nih.gov/articlerender.fcgi?artid=35 90218\&tool=pmcentrez\&rendertype $=$ abstract.

[15] Gumede-moyo S, Filteau S, Munthali T, Todd J, Musonda P. Implementation effectiveness of revised (post-2010) World Health Organization guidelines on prevention of mother-tochild transmission of HIV using routinely collected data in sub-Saharan Africa A systematic literature review. 2017;0 (December 2016). 